\title{
Le spin des électrons se transporte-t-il comme leur charge?
}

Fabian Cadiz, Alistair C.H. Rowe et Daniel Paget (daniel.paget@polytechnique.edu) Physique de la matière condensée (École polytechnique, CNRS, Université Paris-Saclay), École polytechnique, 91128 Palaiseau

\section{Pour répondre aux besoins}

croissants de la microélectronique,

\section{il a été proposé de transmettre}

l'information par le spin

de l'électron, cette variable

quantique dont l'analogue

\section{classique est la rotation}

\section{sur lui-même.}

II serait naturel de penser que

la charge de l'électron et son spin

se transportent de manière

identique, si l'on excepte

la tendance de toute orientation

de spin à disparaitre par relaxation.

Et pourtant, plusieurs études

fondamentales montrent

que le spin peut se transporter

de façon indépendante de l'électron

qui le porte, et donc « vivre sa vie »

comme une particule autonome.

Cet article est consacré

à ces résultats fortement

contre-intuitifs.
Les propriétés de spin des électrons dans les semi-conducteurs sont étudiées de manière fondamentale depuis plusieurs dizaines d'années. Ces études ont trouvé un regain d'intérêt lorsque l'on a imaginé des circuits "spintroniques ", dans lesquels l'information qui se transmet n'est plus portée par l'électron lui-même mais par son spin.

D'un point de vue fondamental, on a cherché à générer des courants électroniques polarisés de spin, c'est-à-dire pour lesquels les électrons de spin $\uparrow$ et $\downarrow$ sont en concentrations différentes, $\mathrm{n}_{\uparrow}$ et $\mathrm{n}_{\downarrow}$ respectivement (encadré 1). Ceci se réalise soit par injection d'électrons polarisés de spin à partir de métaux ferromagnétiques, soit par une excitation lumineuse polarisée circulairement qui, dans certaines conditions, permet de transférer aux photoélectrons le moment angulaire du photon (c'est le pompage optique). On a mesuré le temps caractéristique de la disparition de cette polarisation, appelé le temps de relaxation de spin, ainsi que la distance caractéristique de cette disparition au cours de processus de transport diffusifs ou de dérive dans un champ électrique.

Dans cet article, nous mettons en lumière trois effets originaux qui ont été révélés par ces études : l'effet Hall de spin, la dépendance de la diffusion des électrons avec le spin et la diffusion ambipolaire de spin.

\section{Courant de spin perpendiculaire au courant de charge}

Nous considérons dans un premier temps les courants de charge et de spin, tels que définis dans l'encadré 1 , et montrons qu'ils peuvent être différents et même avoir des directions perpendiculaires l'une à l'autre. L'une des premières démonstrations expérimentales de cette séparation des courants nous a été donnée il y a plus de dix ans par les travaux d'une équipe de l'université de Santa Barbara, en Californie [1]. Suivant les prédictions d'un article théorique de M. I. Dyakonov et V. I. Perel paru trente ans plus tôt, ces chercheurs ont simplement fait passer un courant électronique dans un film semi-conducteur d'arséniure de gallium, le long d'une direction y. Comme les électrons ne sont pas polarisés, le courant de spin le long de y est nul. En utilisant une technique basée sur le changement de l'orientation de la polarisation linéaire d'un faisceau laser sonde (effet Kerr), ils ont mesuré la composante de la polarisation de spin des électrons $(\uparrow$ ou $\downarrow$ ) dans la direction perpendiculaire au plan de l'échantillon. Comme le montre la figure 1c, on observe une accumulation de spin $\uparrow$ sur la gauche de l'échantillon et une accumulation de spin $\downarrow$ sur la droite. Ainsi, tandis que le courant électrique circule le long de y, il s'établit un courant de spin dans la direction perpendiculaire $\mathrm{x}$, qui est responsable de 
cette accumulation. Cet effet est l'analogue de spin de l'effet Hall classique, bien qu'ici aucun champ magnétique ne soit appliqué (voir les schémas a et b de la figure 1), et donc a été appelé effet Hall de spin. Il est dû à l'existence d'un couplage entre l'impulsion de l'électron (son vecteur d'onde k) et son spin : l'interaction spin-orbite, qui a pour origine la précession du spin électronique dans le champ magnétique créé par le mouvement de l'électron. En raison de ce couplage, comme l'illustre le schéma d de la figure 1, dans le cas d'une collision avec une impureté chargée, les électrons de spin $\uparrow$ ont une probabilité légèrement plus forte de diffuser vers la gauche, tandis que c'est l'inverse pour les électrons de spin $\downarrow$. On voit qu'après de multiples collisions de ce type, il apparait bien une séparation des électrons entre la gauche et la droite en fonction de leur spin $\uparrow$ ou $\downarrow$ perpendiculaire à la surface.

De nombreuses études ont mis en évidence cet effet dans différents matériaux, ainsi que l'effet réciproque, pour lequel un courant de spin génère un courant de charge. Cet effet réciproque, l'effet Hall de spin inverse, a été envisagé comme un moyen de détection électrique du spin électronique.
Effet Hall classique
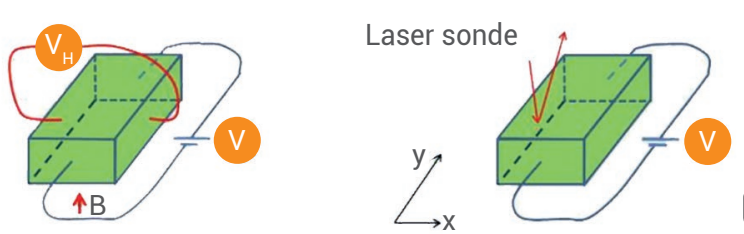

b

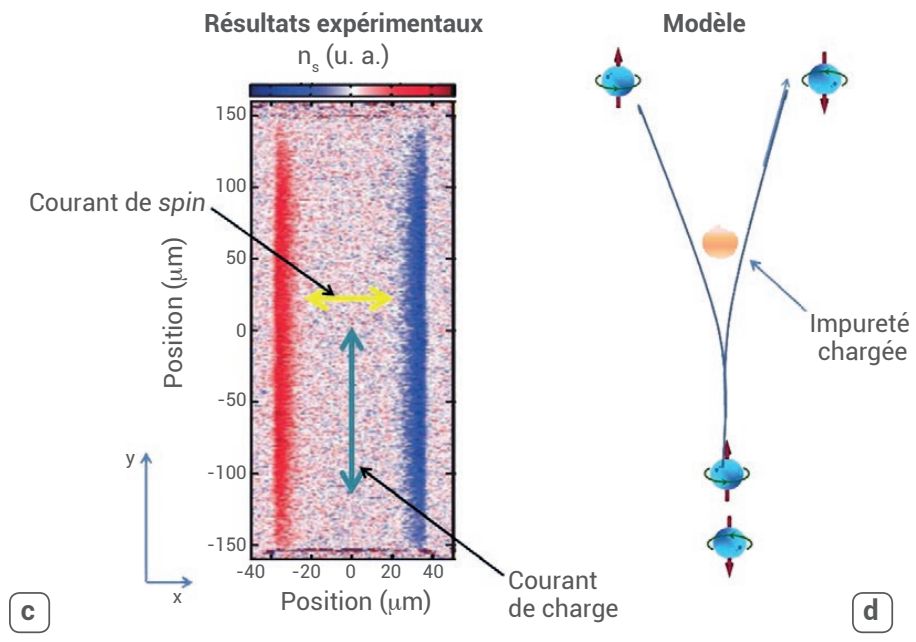

1. Effet Hall de spin.

(a) L'effet Hall classique consiste en l'apparition d'une tension latérale $V_{H}$, lors du passage d'un courant dans un champ magnétique $\mathbf{B}$.

(b) En revanche, pour l'effet Hall de spin, il n'y a pas de champ magnétique appliqué et on sonde par réflexion d'un laser polarisé (effet Kerr) la répartition spatiale des orientations de spin $\uparrow$ ou $\downarrow$ perpendiculaires à la surface de l'échantillon.

(c) On observe alors une accumulation de spins $\uparrow$ à gauche (en rouge) et une accumulation de spins $\downarrow$ à droite (en bleu). Ce résultat montre qu'il apparait un courant de spin le long de $\mathrm{x}$.

(d) Ceci est dû aux collisions dépendant du spin avec les impuretés chargées. Au moment des collisions, les électrons sont déviés préférentiellement vers la gauche ou la droite, en fonction de leur spin ( $\uparrow$ ou $\downarrow)$.

Le spin : une particule indépendante de l'électron?

Pour des électrons polarisés de spin, et une fois choisi l'axe de quantification du spin, la population d'électrons $\left(n=n_{\uparrow}+n_{\downarrow}\right)$ se sépare en une sous-population de spin $\uparrow$ et de concentration $\mathrm{n}_{\uparrow}$, et une sous-population de spin $\downarrow$ et de concentration $\mathrm{n}_{\downarrow}$ avec une densité de spin $s=n_{\uparrow}-n_{\downarrow}$, et une polarisation de spin $\mathrm{s} / \mathrm{n}$.

Le transport de spin (s) et celui de la charge (n) sont définis par des grandeurs spécifiques bien distinctes. Sous champ électrique, alors que le courant électronique est relié à la vitesse $v_{\uparrow}\left(v_{\downarrow}\right)$ des spins $\uparrow(\downarrow)$ par $J=n_{\uparrow} e v_{\uparrow}+n_{\downarrow}$ ev $v_{\downarrow}$ (où e est la charge de l'électron), le courant de spin est donné par $J_{s}=n_{\uparrow} e v_{\uparrow}-n_{\downarrow} e v_{\downarrow}$. Si $\tau$ est le temps de vie de l'électron, dû principalement à la recombinaison avec un trou, le temps de vie de spin $\tau_{s}$ est en général plus court que $\tau$, en raison des transitions (de type $\uparrow \leftrightarrow \downarrow$ ) entre les deux types de spin qui sont responsables de la relaxation de spin.
Pour un transport diffusif, c'est-à-dire à champ électrique nul, la loi de Fick relie le courant au gradient spatial de concentration. On écrit cette loi séparément pour les deux spins :

$\mathrm{J}_{\uparrow}=e \mathrm{D}_{\uparrow} \nabla \mathrm{n}_{\uparrow}$ et $\mathrm{J}_{\downarrow}=e \mathrm{D}_{\downarrow} \nabla \mathrm{n}_{\downarrow}$,

où $\mathrm{D}_{\uparrow(\downarrow)}$ est la constante de diffusion. Par différence, compte tenu de $\nabla \mathrm{n}=\nabla \mathrm{n}_{\uparrow}+\nabla \mathrm{n}_{\downarrow}$ et $\nabla \mathrm{s}=\nabla \mathrm{n}_{\uparrow}-\nabla \mathrm{n}_{\downarrow}$, on obtient :

$J_{s}=J_{\uparrow}-J_{\downarrow}=e\left[\left(D_{\uparrow}+D_{\downarrow}\right) / 2+\left(D_{\uparrow}-D_{\downarrow}\right) \nabla n / 2 \nabla s\right] \nabla s$,

ce qui permet de définir une constante de diffusion de spin effective :

$D_{s}=\left(D_{\uparrow}+D_{\downarrow}\right) / 2+\left(D_{\uparrow}-D_{\downarrow}\right) \nabla n / 2 \nabla s$.

Si $D_{\uparrow}=D_{\downarrow}$, on a $D_{s}=D$ (la constante de diffusion de la charge électronique). Dans le cas inverse, la diffusion de spin dépend du gradient spatial de charge et on a $D_{s} \neq D$ : il apparait alors un couplage charge-spin. 
Comment étudier séparément les diffusions
de charge et de spin?

Pour étudier la diffusion de spin, nous utilisons une expérience de microluminescence polarisée (fig. 2a). Nous générons de façon très localisée dans un échantillon semi-conducteur des photoélectrons polarisés de spin par une excitation laser polarisée circulairement. Nous analysons sur le détecteur CCD le profil spatial de la luminescence, proportionnelle à la somme des concentrations des électrons de spin $\uparrow$ (majoritaires) et des électrons de spin $\downarrow$ (minoritaires). Comme le montre la figure $2 \mathrm{~b}$, on observe un profil dont l'extension spatiale est plus grande que la tache du faisceau laser, en raison de la diffusion des photoélectrons après leur création. Pour étudier la diffusion du spin électronique, on analyse la différence $\mathrm{I}\left(\sigma^{+}\right)-\mathrm{I}\left(\sigma^{-}\right)$des composantes de la luminescence d'hélicités $\sigma^{+}$et $\sigma^{-}$. Cette grandeur est directement liée à l'orientation de $\operatorname{spin} \mathrm{s}=\mathrm{n}_{\uparrow}-\mathrm{n}_{\downarrow}$. Les distances caractéristiques sur lesquelles on peut conserver la charge et le spin dans un mouvement diffusif sont définies par les longueurs de diffusion de charge $\mathrm{L}=(\mathrm{D} \tau)^{1 / 2}$ et de $\operatorname{spin} \mathrm{L}_{\mathrm{s}}=\left(\mathrm{D}_{\mathrm{s}} \tau_{\mathrm{s}}\right)^{1 / 2}$. Leur mesure, couplée à celles des temps de vie de charge $\tau$ et de $\operatorname{spin} \tau_{\mathrm{s}}$ par luminescence résolue en temps, permet de remonter aux constantes de diffusion $\mathrm{D}$ et $\mathrm{D}_{\mathrm{s}}$ de charge et de spin. Une illustration de cette technique est donnée dans la figure 2c, qui montre le profil de charge en champ électrique nul et pour un champ électrique de $400 \mathrm{~V} / \mathrm{cm}$. Tandis que, dans le premier cas, le profil est spatialement isotrope, l'application du champ électrique montre la dérive des électrons dans ce champ [2].

L'une des techniques les plus directes, bien que relativement moins sensible que la technique de microluminescence polarisée, pour mesurer la constante de diffusion de spin $\mathrm{D}_{\mathrm{s}}$ est celle du réseau de spin. Elle consiste à faire interférer à la surface de l'échantillon des impulsions laser polarisées linéairement à $90^{\circ}$ l'une de l'autre (fig. 3a). Cette technique élégante permet de créer un profil de franges d'interférences, non pas de l'intensité mais de l'hélicité de la lumière. Ainsi, on génère un profil d'interférence de la densité de spin s (fig. 3b), tandis que la concentration de charge $\mathrm{n}_{\uparrow}+\mathrm{n}_{\downarrow}$ est homogène dans l'espace. Il n'y a donc pas de courants de charge, et l'étude de la diffraction d'un faisceau sonde permet de détecter uniquement la dynamique des courants de spin (fig. 3c). Cette technique a été utilisée par une collaboration de laboratoires californiens pour montrer que l'on peut avoir $\mathrm{D}_{\mathrm{s}}<\mathrm{D}$ (fig. 3d) [3].
En effet, il existe des collisions qui affectent le courant de spin sans affecter le courant de charge, comme celle qui est représentée en haut à droite de la figure $3 \mathrm{~d}$, et qui consiste en un retournement simultané des spins de deux électrons, conséquence de leur interaction d'échange.

\section{Pourquoi la diffusion de l'orientation de spin peut-elle différer de celle des électrons eux-mêmes ?}

L'encadré 1 (p. 7) montre que la diffusion de l'orientation de spin peut différer de celle de la charge. L'hypothèse principale est que les processus de transition entre les deux états de spin ne sont pas très rapides, sinon, au moins à champ magnétique nul, on ne pourrait pas établir de polarisation de spin. On peut donc considérer séparément les électrons de spin $\uparrow$ et ceux de spin $\downarrow$ comme deux populations distinctes obéissant chacune à sa propre statistique, et donc ayant chacune sa propre constante de diffusion, que nous appellerons $\mathrm{D}_{\uparrow}\left(\mathrm{D}_{\downarrow}\right)$. Dans ce cas, on peut avoir $\mathrm{D}_{\mathrm{s}} \neq \mathrm{D}$, pourvu que l'on ait $\mathrm{D}_{\uparrow} \neq \mathrm{D}_{\downarrow}$.

Nous allons présenter deux phénomènes physiques distincts qui entraînent $\mathrm{D}_{\uparrow} \neq \mathrm{D}_{\downarrow}$. Ils surviennent tous deux à forte concentration électronique.
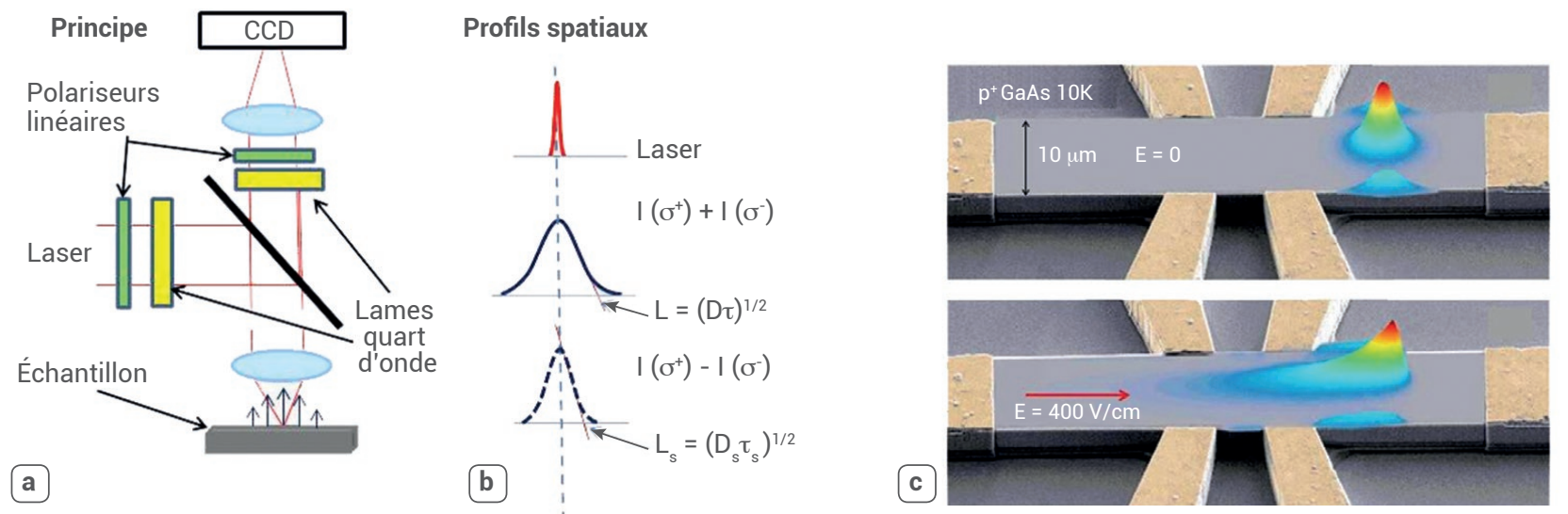

2. Microluminescence polarisée.

(a) On focalise le laser sur l'échantillon à la limite de diffraction. À l'aide de lames quart d'onde placées sur le faisceau laser et sur le faisceau de luminescence, on polarise circulairement le laser et on mesure sur le détecteur CCD les deux composantes de la luminescence, d'hélicités $\sigma^{+}$et $\sigma^{-}$. (b) Le profil spatial de leur somme, proportionnelle à la concentration de photoélectrons, s'étend au-delà de celui du laser. La dépendance spatiale de ce profil donne la longueur de diffusion de charge $L$, qui dépend de la constante de diffusion $D$ et du temps de vie de charge $\tau$. Le profil spatial de leur différence, qui est proportionnelle à l'orientation de $\operatorname{spin} s=n_{\uparrow}-n_{\downarrow}$, donne la longueur de diffusion de $s p i n L_{s}$, qui dépend de la constante de diffusion de spin $\mathrm{D}_{\mathrm{s}}$ et du temps de vie de $\operatorname{spin} \tau_{\mathrm{s}}$

(c) Profils de concentration des photoélectrons dans le cas d'un barreau de GaAs, avec des contacts électriques sans (image du haut) et avec (image du bas) champ électrique appliqué. Si l'on applique un champ électrique, il apparait une queue liée à la dérive des photoélectrons dans ce champ. 


\section{La diffusion des électrons dépend de leur spin : une conséquence du principe de Pauli}

Si l'on applique les deux techniques de microluminescence polarisée et de réseau de spin décrites ci-dessus à un film de GaAs à basse température, on observe à basse puissance lumineuse que la polarisation $\mathrm{s} / \mathrm{n}$ décroit lorsque l'on s'éloigne de la position du faisceau laser (fig. 4a). Cette décroissance reflète la disparition du spin par relaxation (encadré 1) pendant la diffusion. En revanche, à forte puissance lumineuse, on observe un profil spatial en forme de volcan (fig. 4b).

Ce profil de type volcan s'interprète par une constante de diffusion des spins majoritaires $(\uparrow)$ supérieure d'environ $50 \%$ à celle des spins minoritaires $(\downarrow)$. En effet, la diffusion centrifuge dépendant du spin cause un appauvrissement sélectif en électrons majoritaires à l'emplacement de la tache du laser et donc une diminution de la polarisation de spin à cet endroit (fig. 4c). Ce résultat est une conséquence directe du principe de Pauli (voir l'encadré 2). a
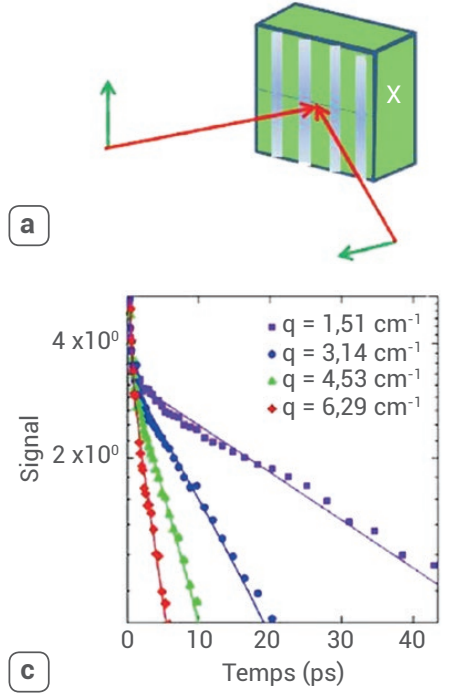

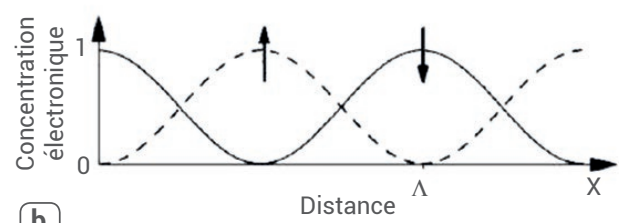

b

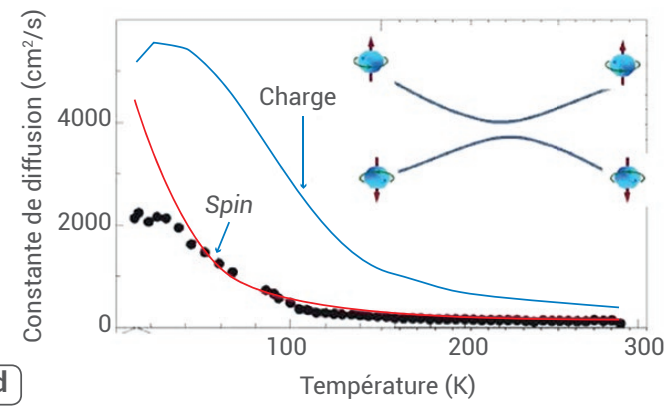

3. Mesure de la constante de diffusion de spin par la technique de réseau de spin.

(a) À l'aide de deux faisceaux laser en impulsion (en rouge), polarisés linéairement, on génère dans l'échantillon un système de franges d'interférences, dont le pas est $\Lambda=1 / q$.

(b) Dépendance spatiale des concentrations $n \uparrow$ et $n \downarrow$ dans l'échantillon : on a créé de manière transitoire un réseau de spin, dont le pas $\wedge$ est déterminé par l'angle d'incidence des faisceaux.

(c) Un faisceau sonde, diffracté par l'échantillon, permet de détecter ce réseau de spin. La mesure au cours du temps de l'intensité du signal réfléchi pour plusieurs valeurs de q (le module du vecteur de diffusion du réseau de spin) permet de remonter à la constante de diffusion de $\operatorname{spin} \mathrm{D}_{\mathrm{S}}$.

(d) Les points noirs montrent la dépendance en température de la constante de diffusion de spin mesurée expérimentalement, tandis que les courbes indiquent les prédictions théoriques. On voit que la constante de diffusion de spin est inférieure à celle de la charge (courbe bleue).

\section{Pourquoi la diffusion des électrons peut dépendre de leur spin à forte concentration ?}

Encadré 2

Pour exprimer la valeur de la constante de diffusion, on utilise une approche thermodynamique qui définit pour une concentration électronique donnée l'énergie de Fermi (ou potentiel chimique) $E_{F}$, qui est, au moins à température nulle, l'énergie jusqu'où les niveaux électroniques sont remplis. La diffusion survient lorsque la concentration électronique $n_{\uparrow}$ ou $n_{\downarrow}$ dépend de la position, et est par exemple plus grande à la position 1 qu'à la position 2, proche de la première (voir l'encart de la figure E1). Dans ces conditions, l'énergie de Fermi en position 1, $\mathrm{E}_{\mathrm{F} 1}$, est supérieure à celle de la position $2, \mathrm{E}_{\mathrm{F} 2}$. Le courant de diffusion $J_{\uparrow}$ (ou $J_{\downarrow}$ ) est alors proportionnel à $E_{F 1}-E_{F 2}$ et va, en raison du signe négatif de la charge électronique, de la position 2 à la position 1. On montre alors, en faisant tendre vers zéro la distance entre ces deux positions, que le courant et donc la constante de diffusion $\mathrm{D}$ sont proportionnels à la dérivée de l'énergie de Fermi par rapport à la concentration électronique, $\partial \mathrm{E}_{\mathrm{F}} / \partial \mathrm{n}$.

Sur la figure E1, la courbe bleue décrit la dépendance en concentration de $E_{F}$, tandis que la courbe rouge montre celle de la constante de diffusion $D$ dans GaAs à $50 \mathrm{~K}$. Tant que la concentration électronique est inférieure à $10^{16} \mathrm{~cm}^{-3}$, le niveau de Fermi est plus bas que le bas de la bande de conduction $\left(E_{F}<0\right.$ ), avec $\partial E_{F} / \partial n$ et donc $D$ indépendants de $n$ (nous prendrons $D=1)$. Pour des concentrations supérieures à $10^{16} \mathrm{~cm}^{-3}$, on a $E_{F}>0$ : on dit que le gaz d'électrons est dégénéré. $\partial E_{F} / \partial n$ et donc $D$ augmentent alors fortement avec $n$.
Ce raisonnement peut s'appliquer séparément aux électrons majoritaires $\left(\mathrm{n}_{\uparrow} \approx 10^{17} \mathrm{~cm}^{-3}\right)$ et aux électrons minoritaires $\left(\mathrm{n}_{\downarrow} \approx 2 \times 10^{16} \mathrm{~cm}^{-3}\right)$. On voit que, dans le régime dégénéré, les constantes de diffusion des spins majoritaires et minoritaires peuvent être significativement plus grandes que leurs valeurs à basse concentration, tout en étant très différentes entre elles, avec $D_{\uparrow}>D_{\downarrow}$.

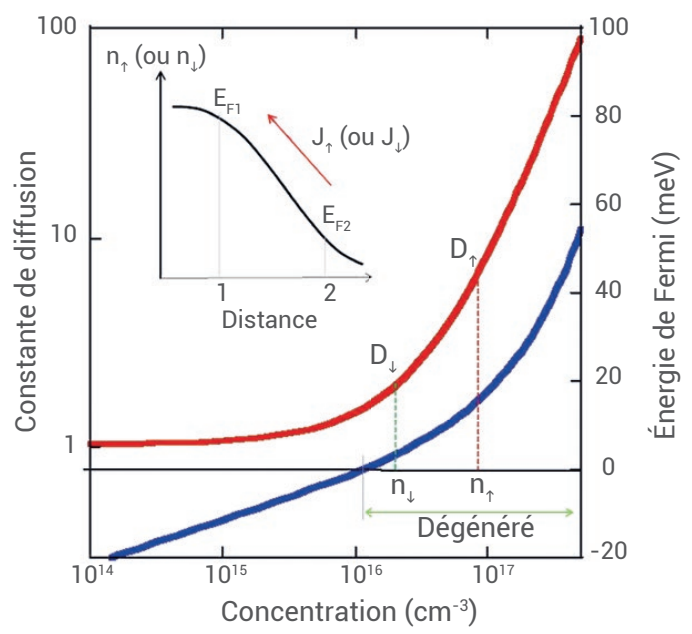

E1. Variations de l'énergie de Fermi (en bleu) et de la constante de diffusion $D$ (en rouge) avec la concentration électronique. 

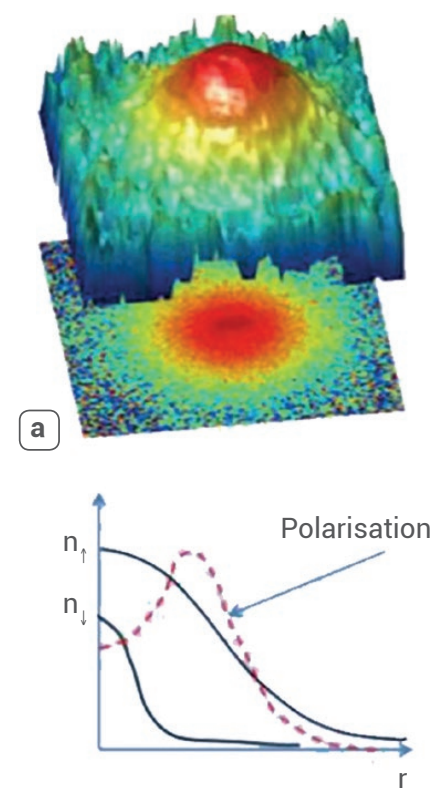

c
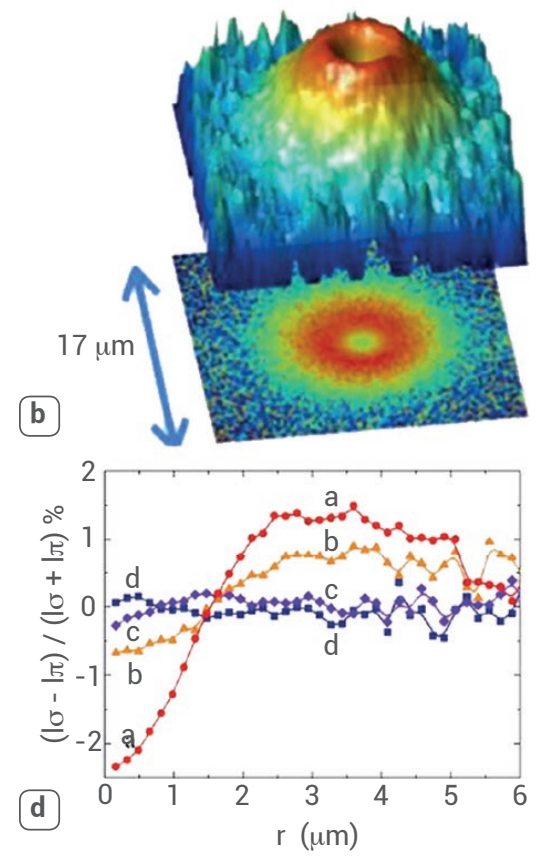

4. Diffusion dépendant du spin dans un gaz de photoélectrons dégénérés.

(a) Profil spatial de la polarisation des photoélectrons à faible puissance lumineuse. Sa décroissance avec la distance reflète la perte de polarisation par relaxation de spin lors de la diffusion.

(b) Profil spatial à forte puissance lumineuse. L'apparition d'un creux au centre du profil montre que la diffusion dépend du spin, et est plus rapide pour les électrons majoritaires $(\uparrow)$ que pour les minoritaires $(\downarrow)$.

(c) Il se produit alors au centre un effet de filtre à spin, qui évacue prioritairement les électrons majoritaires, et donc diminue la polarisation de spin à cet endroit.

(d) Différence relative entre les profils de l'intensité de luminescence, qui reflètent ceux de la concentration électronique $n_{\uparrow}+n_{\downarrow}$, pour une excitation polarisée circulairement ( $\sigma$, électrons polarisés de spin), et pour une excitation polarisée linéairement ( $\pi$, électrons non polarisés). On observe, à forte puissance lumineuse (courbe a), un signal de luminescence plus faible au centre en excitation $\sigma$, compensé par un excès de signal à une certaine distance $(r \approx 2$ à $5 \mu \mathrm{m})$. Ceci signifie que la diffusion des électrons, moyennée sur le spin, est légèrement plus rapide lorsqu'ils sont polarisés de spin que dans le cas contraire. Cet effet de la polarisation de la lumière disparait lorsque l'on diminue la puissance lumineuse (courbes successives b, c, et d).

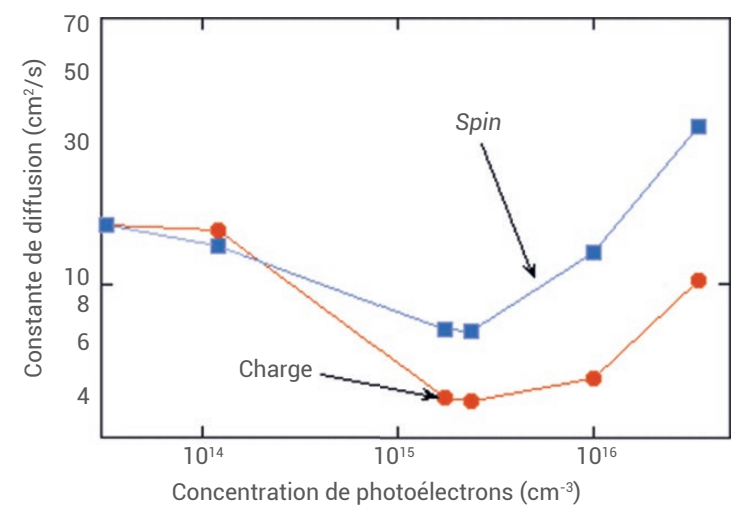

5. Valeurs des constantes de diffusion de charge et de spin dans GaAs à $15 \mathrm{~K}$, en fonction de la concentration de photoélectrons. Pour une concentration supérieure à $10^{16} \mathrm{~cm}^{-3}$, ces résultats ont été obtenus par réseau de spin (fig. 3). Pour des concentrations plus faibles, cette dernière technique n'étant pas utilisable pour des raisons de sensibilité, nous avons mesuré l'extension caractéristique des profils spatiaux de charge et de polarisation et obtenu $D$ et $D_{s}$ à partir des longueurs de diffusion de charge et de spin (fig. 2). Le ralentissement de la diffusion de charge quand $\mathrm{n}$ augmente de $10^{14}$ à $10^{15} \mathrm{~cm}^{-3}$ est dû aux effets électrostatiques liés aux trous (diffusion ambipolaire, voir encadré 3 ), et affecte plus la charge que le spin. En conséquence, la constante de diffusion de spin est supérieure à celle de la charge dans toute la gamme de concentrations électroniques. Pour $\mathrm{n}>10^{16} \mathrm{~cm}^{-3}$, on observe une augmentation des deux constantes de diffusion, liée au principe de Pauli (encadré 2).
Dans ces conditions, on peut montrer que la constante de diffusion $\langle\mathrm{D}\rangle$ de la charge électronique, c'est-à-dire la moyenne pondérée des constantes de diffusion des deux espèces de spin, est plus élevée lorsque les électrons sont polarisés de spin que lorsque la polarisation de spin est nulle, ce qui se réalise par une excitation lumineuse de même puissance, mais polarisée linéairement. Comme le montre la figure $4 \mathrm{~d}$, on observe à forte puissance lumineuse une différence relative, de l'ordre de quelques pour cents, entre les deux profils de l'intensité de luminescence. Cet effet, du deuxième ordre en fonction de la polarisation de spin, est apparenté à la pression de dégénérescence quantique, qui empêche par ailleurs les étoiles de s'effondrer sur elles-mêmes sous l'effet de la seule gravitation (un tel effondrement impliquerait que des électrons occupent le même état quantique, ce qui est contraire au principe de Pauli). Cette observation indique que, conformément à la théorie, la pression de dégénérescence dépend du spin et chasse plus facilement les électrons du centre lorsqu'ils sont polarisés de spin que dans le cas inverse.

\section{L'orientation de spin ne diffuse pas comme la charge}

Nous avons combiné les techniques de réseau de spin et de microluminescence polarisée décrites plus haut pour mesurer $D_{\mathrm{s}}$, dans une vaste gamme de concentrations électroniques. Comme le montre la figure 5, outre l'augmentation des constantes de diffusion qui survient à très forte concentration en raison du principe de Pauli (encadré 2), on obtient des rapports $D_{\mathrm{s}} / D$ supérieurs à 2 dans une vaste plage de concentrations [4]. Ceci signifie que l'orientation de spin $s$ décroit moins vite avec la distance que la concentration. Il faut bien sûr pour cela que la distance à la tache laser ne soit pas trop grande pour qu'il reste des électrons.

Il s'agit là d'un effet électrostatique dans un système à trois composants (diffusion ambipolaire de spin) : les électrons des deux spins, et les trous qui diffusent plus lentement [5]. Comme l'illustre l'encadré 3, l'interaction électrostatique entre trous et électrons de spin $\uparrow$ d'une part et électrons de spin $\downarrow$ d'autre part, permet d'établir un couplage spin-spin entre les deux types d'électrons. L'encadré 1 montre que, dans 


\section{Pourquoi le spin peut-il diffuser plus vite que la charge?}

La figure E2a représente la répartition spatiale des deux types de spins électroniques (en bleu) et des trous (en orange) à l'instant de leur création par la lumière. Tous ces porteurs se trouvent dans la zone de focalisation du laser, dont le centre est indiqué par la ligne verticale. Le régime stationnaire s'établit en tenant compte de ce que les trous, de charge électrique + , diffusent moins que les électrons, et du fait que les spins électroniques $\uparrow$ sont plus nombreux que les $\downarrow$.

La diffusion des spins $\uparrow$ crée un champ électrique interne $E_{+}$, qui ramène les électrons au centre et tend à rétablir en tout point la neutralité électrique, tandis que la diffusion des spins $\uparrow$ crée un champ électrique interne plus faible $E_{\text {_. }}$ Or chaque espèce de spin « ressent » le champ électrique somme $E_{+}+E_{2}$. Ainsi les spins $\downarrow$ sont ramenés au centre par un champ électrique bien plus fort que le seul champ $E_{-}$qu'ils ont créé. On peut comprendre ainsi qu'en régime stationnaire, après diffusion (fig. E2b), les électrons de spin minoritaire ont moins diffusé que les majoritaires (dans cette illustration, ils sont confinés à l'intérieur des courbes pointillées), c'est-à-dire que l'orientation de spin s a diffusé plus loin que la charge $n$.

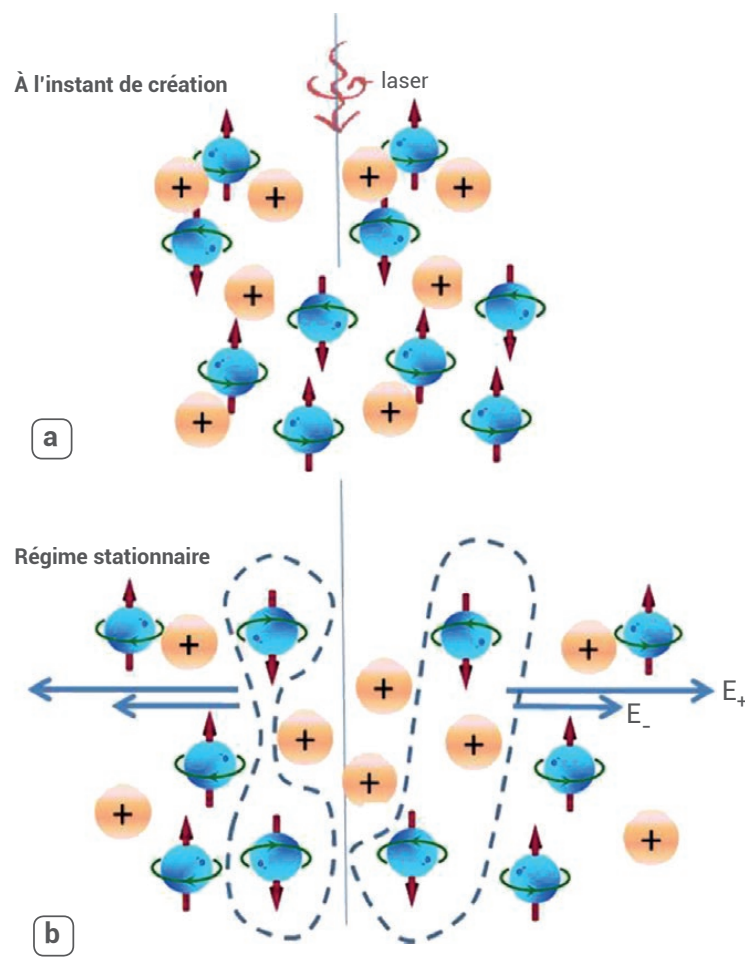

E2. Répartition spatiale des spins électroniques $\uparrow$ et $\downarrow$ et des trous créés par un faisceau laser dans un semi-conducteur. ce cas, il apparait une différence entre $D$ et $D_{\mathrm{s}}$, avec de façon plus précise $D_{\mathrm{s}}>D$. Ainsi, le ralentissement de la diffusion des électrons à cause des trous affecte moins le spin que la charge.

\section{Conclusion}

Cet article donne plusieurs illustrations de l'interdépendance des propriétés de transport de charge et de spin dans les semi-conducteurs. Dans la quasi-totalité des exemples, cette interdépendance tire son origine de l'existence de couplages, charge-spin (effet du principe de Pauli), spin-spin (diffusion ambipolaire), ou spinorbite (effet Hall de spin). Bien que chaque électron porte effectivement son spin et se transporte avec lui, ce sont dans les propriétés moyennes sur un grand nombre d'électrons qu'apparaissent ces effets, à condition de séparer les électrons en deux espèces indépendantes, chacune caractérisée par son état de spin.

Ce domaine reste très actif, car d'autres effets ont été prédits et restent à observer. Par exemple, il est connu que la bande interdite d'un semi-conducteur dépend des charges libres qui s'y trouvent. En raison de ce mécanisme, l'énergie de chaque type d'électron, et donc la constante de diffusion, peut dépendre de la concentration de l'autre type, ce qui génère un mécanisme original de couplage charge-spin et spin-spin. De plus, les effets attendus pourraient être très différents pour des systèmes semi-conducteurs de dimensionnalité réduite comme les puits quantiques, les nanofils ou les matériaux bidimensionnels tels que les dichalcogénures de métaux de transition...

\section{Références}

1• Y. K. Kato et al., "Observation of the Spin Hall Effect in Semiconductors", Science 306 (2004) 1910-1913.

2• F. Cadiz et al., "Absence of carrier separation in ambipolar charge and spin drift in p+-GaAs", Appl. Phys. Lett . 107 (2015) 162101.

3. C. P. Weber et al., "Observation of spin Coulomb drag in a two-dimensional electron gas", Nature 437 (2005) 1330.

4• F. Cadiz et al., "Ambipolar spin diffusion in p-type GaAs: a case where spin diffuses more than charge", J. Appl. Phys. 122 (2017) 095703.

5• F. Cadiz et al., "Ambipolar spin-spin coupling in p+ GaAs", Phys. Rev. B 92 (2015) 121203 (R). 